\title{
Chronic inflammation as a link between periodontitis and secondary amyloidosis: A case report and mini-review
}

\author{
Özden Kanbolat ${ }^{1}$, Kuddusi Cengiz ${ }^{*}$, Umur Sakallı², Elif Dağıstan ${ }^{1}$, Ahmet Öztürk ${ }^{3}$, Fatma Kutsal and Bilge Can $^{3}$ \\ ${ }^{1}$ Department of Nephrology, Samsun Education and Research Hospital, Samsun Ondokuz Mayıs University, Turkey \\ ${ }^{2}$ Department of Periodontology, Samsun Education and Research Hospital, Samsun Ondokuz Mayıs University, Turkey \\ ${ }^{3}$ Department of Pathology, Samsun Education and Research Hospital, Samsun Ondokuz Mayıs University, Turkey
}

\begin{abstract}
Background: Periodontitis may be an important occult source of chronic inflammation that increases the acute-phase reactant levels in patients and hence, might affect the development of amyloidosis. Deposition of amyloid fibrils derived from circulating acute-phase reactant serum amyloid A protein causes systemic amyloidosis, a serious inflammatory disorder.
\end{abstract}

Study design and methods: We document a male patient who developed reactive amyloidosis (AA type), most likely secondary to his longstanding periodontitis. A careful dental and periodontal examination was performed. Acute-phase reactants were measured at baseline and at 4 to 6 weeks after completion of periodontal therapy. Intra-oral and renal biopsies were performed.

Results: The mean probing depth was $8.20 \pm 0.74 \mathrm{~mm}$. The serum inflammatory markers improved more dramatically at the second week of periodontal therapy than any other time intervals.

Conclusion: Periodontitis may be an additive factor to traditional etiological factors for systemic AA amyloidosis as well as being a primary etiological factor. There, treating or preventing periodontal diseases may alleviate disease burden or may even prevent the development of systemic amyloidosis.

\section{Introduction}

Reactive systemic AA amyloidosis, with a sustained acutephase response can complicate chronic inflammatory disorders. AA amyloid fibrils are derived from the acute-phase reactant (APR) serum amyloid A protein (SAA) through a process of cleavage, mis holding, and aggregation [1]. Renal disease is a frequent manifestation of the systemic amyloidosis and a major cause of morbidity [1]. SAA is an apolipoprotein constituent of high-density lipoprotein that is synthesized by the hepatocytes under the transcriptional regulation of pro-inflammatory cytokines [2]. The activation pattern of SAA protein in the presence of inflammation is similar to that of $\mathrm{C}$-reactive protein (CRP). SAA and CRP are both termed first-class acute-phase reactants (APRs) since they are the most sensitive plasma proteins indicating inflammatory activity [3,4]. Sustained overproduction of SAA is prerequisite for the development of systemic AA amyloidosis.

Secondary amyloidosis, representing approximately $45 \%$ of all cases of systemic amyloidosis, has been associated with various chronic inflammatory conditions such as rheumatoid arthritis, sarcoidosis, Crohn's disease, ulcerative colitis and tuberculosis. Secondary amyloidosis has also been linked to malignant diseases such as Hodgkins's disease and mesothelioma. In addition, familial Mediterranean fever (FMF), an autosomal recessive disease, primarily affects the population in the Mediterranean basin [1]. FMF seems to be the leading cause of AA amyloidosis in Turkey, followed by chronic inflammatory diseases and unknown causes [1,5-9]. However, in up to approximately $42 \%$ of cases of AA amyloidosis no underlying etiology can be identified. The progression of secondary amyloidosis depends on the nature and status of the underlying chronic inflammatory disease. For example, secondary amyloidosis -associated tuberculosis has been shown to undergo remission when the chronic infection has been eliminated [10]. It has been shown that patients with chronic periodontitis display signs of a subclinical systemic inflammatory condition [11]. Furthermore, treatment of advanced periodontitis by full-mouth tooth extraction reduced systemic levels of cardiovascular risk and inflammatory reaction [12].

Cross-sectional studies have demonstrated that plasma levels of inflammatory markers such as CRP, fibrinogen, IL-6 and leukocyte counts increase in periodontitis patients when compared to periodontally healthy patients $[13,14]$. Some studies have shown that effective periodontal therapy reduced levels of CRP [14]. This implies that inflammatory reaction triggered by periodontitis contributes to the whole-body inflammatory burden. Also, these inflammatory markers have been reported to be robust predictors of AA amyloidosis [1]. It has been reported that serum APR levels are increased in periodontal and systemic AA amyloidosis patients [15-17]. However, APRs that have increased during periodontal disease play on important role as an etiopathological factor for systemic AA amyloidosis. It is also known that chronic infection or inflammatory disease may

${ }^{\star}$ Correspondence to: Kuddusi Cengiz, Department of Nephrology, Samsun Education and Research Hospital, Samsun Ondokuz Mayıs University, Turkey; E-mail: kcengiz@omu.edu.tr

Key words: periodontitis, inflammation, secondary aa amyloidosis

Received: November 01, 2020; Accepted: December 08, 2020; Published: December 11, 2020 
cause systemic AA amyloidosis, even without obvious infection or inflammation $[18,19]$.

\section{Periodontal disease shares several clinical and etiopathogenic characteristics with AA amyloidosis}

In addition to the fact that localized deposits of amyloid in patients with systemic AA amyloidosis can aggravate periodontal disease, chronic periodontal disease may exaggerate AA amyloidosis via increased levels of systemic inflammatory mediators. Indeed, patients with chronic periodontal disease have higher levels of SAAthe precursor protein of amyloid fiber in AA amyloidosis than patients without periodontal disease [20]. Therefore, elimination of the local infection associated with periodontal diseases will aid in reducing levels of systemic inflammatory mediators, which may slow the progression of AA amyloidosis [1,10,15-17,21].

The systemic immune response, genetic and environmental factors also effect the risk of developing periodontitis and amyloidosis $[22,23]$. It has been reported that SAA and others APRs were elevated in response to an intravenous challenge with live Porphyromonas gingivalis (P.gingivalis), an important periodontal pathogen [24]. A limited number of experimental studies have shown that SAA and other APRs were elevated in response to an intravenous challenge with P.gingivalis [25]. SAA and CRP concentrations in patients with chronic periodontitis are comparably elevated. High serum titers of antibodies to $P$. Gingivalis and the presence of periodontal disease are independently related to high SAA and CRP levels [26]. Lastly, serum APRs that increase during periodontal disease contribute to the etiopathogenesis of systemic AA amyloidosis. So, the current evidence is such that prevention and treatment of periodontal disease may reduce chronic systemic disease risk at both the individual and community level.

\section{Case presentation}

Our patient is 28 year-old Turkish male, a primary school graduate and who lives in a rustic area. He was unskilled worker and nonsmoker. He was fully informed about the study and written consent was obtained from him prior to examination. In his medical examination he explained that his gums started bleeding at a very early age. At age 12 , he started to experience difficult in eating and complained of tooth mobility and gum bleeding. He claimed that his teeth ached a lot and as a result the teeth were extracted by a non-dentist or himself. Consequently, at the age of 20 to 28 , he lost his three teeth. At age 26, he was diagnosed with periodontitis. Nonetheless, he had not seen a dentist for his problem or performed any personal oral hygiene. He claimed to have had great deal of trouble in his lifetime with his teeth or gums. He reported using various analgesic medications to treat headache of facial pain daily or weekly. Furthermore, he experienced bleeding while brushing his teeth, had tooth mobility and had tooth recession. So, he claimed to brush his teeth 1-2 times a month. The patient complained of pain in his oral cavity (burning mouth) especially on the tongue, buccal mucosa, and had difficulty chewing and swallowing solid foots for three months. The patient presented with periorbital edema, as well as edema in his abdomen and lower extremities. He had been prescribed and oral dose of furosemide $60 \mathrm{mg}$ daily for his anasarca by a family physician at a clinic five days prior. The patient presented with nephrotic syndrome (gross proteinuria, hypoalbuminemia, hyperlipidemia and peripheral edema). The patient referred to us for kidney biopsy. His past medical history was significant for a tonsillectomy as a child. All the symptoms in his medical reviews were negative. Spirometric pulmonary tests, high resolution computed tomography (HRCT), magnetic resonance imaging of the tongue revealed no sign of abnormality. Head and neck examinations were normal. He underwent extensive tests to identify etiologies associated with systemic amyloidosis. Tests such as echocardiogram, abdomen ultrasonography abdomen tomography, serum and protein electrophoresis as well as liver, thyroid function test were all normal. Only moderately diffuse hepato-splenomegaly and enlarged appendix were detected in both abdomen ultrasonography and tomography. Clinical examination revealed poor oral hygiene and heavy plaque accumulation. The tongue was diffusely enlarged (macroglossia) and clear red appearance and bilateral white plaques bleeding easily by gentle removing, and irregular translucent papules and moderately alveolar bone lost were present (Figures 1 and 2). Based upon our patient's history, these papules developed spontaneously or after minor trauma. The enlarged tongue has interfered with speech and swallowing and caused sleep apnea. In addition, three profoundly painful, large ulcers with erythematous halos and a dirty-white ulcer bed were detected, localized on the right and left buccal mucosa. Cultures from the plaques on the tongue and oral cavity were all negative for bacteria and fungi. The biopsies were obtained from the tongue, buccal mucosa, retromolar trigon and kidney. Secondary amyloidosis (AA type) was diagnosed by histological and immunohistochemical findings (Figure 3). Despite amyloid material was seen in the gingival biopsy, unfortunately because of technical lack, it couldn't demonstrate.

Our patient received comprehensive periodontal therapy, which included careful oral hygiene instructions, curettage combined with non-surgical and surgical therapy. Serum inflammatory markers and some laboratory data improved dramatically at the second week of the periodontal therapy more than at any other time interval (Table 1). Restorative treatment started after his periodontal condition was stabilized.

After five days of the medical treatment his edema was dissolved, general health getting little beat well. Unfortunately, we lost the patient on the twelth week of the follow-up.

\section{Discussion}

Our patient was 28-year-old male, a primary school graduate, unskilled worker and non-smoker. He had low income (less than 350 dollar per month). He had not visited any dental service or a dentist regularly. Only he had visited a dentist in emergency condition. He suffered severe chronic periodontitis and presented with nephrotic syndrome. Our patient complained of pain in the oral cavity especially on the tongue, buccal mucosa and had difficulty in chewing and swallowing solid foods for almost three months. The kidney and oral cavity biopsies were performed, and secondary amyloidosis was diagnosed based upon histological and immunohistochemical findings.

Our patient presented with typical signs of periodontal disease that include gingival tenderness, bleeding, recession, alveolar bone

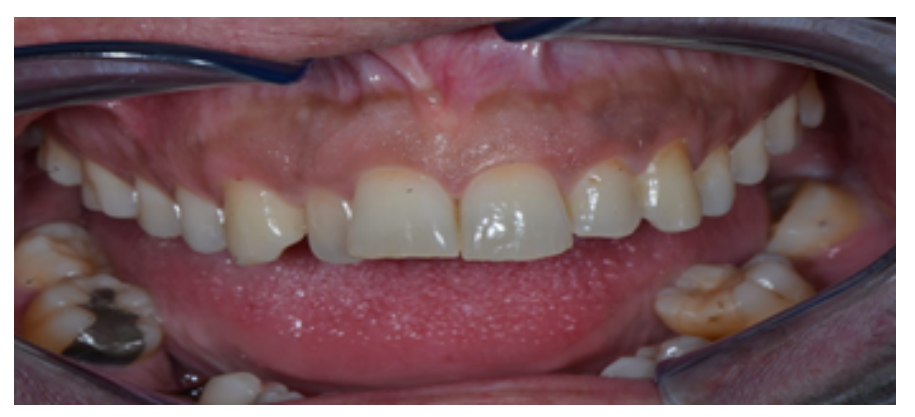

Figure 1. The tongue was diffusely enlarged (macroglossia) 


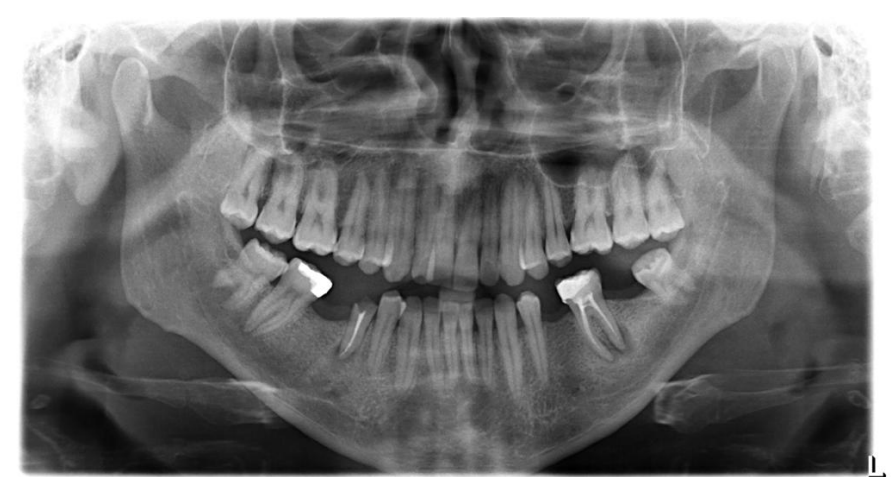

Figure 2. Radiographic findings from the patient described in this case report moderate osteopeny in mandibular and light prominence trabeculation of maxilla are present

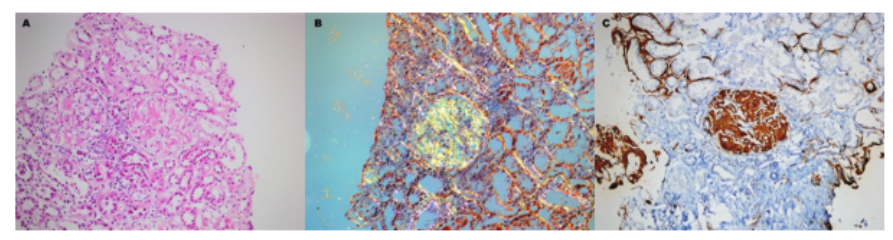

Figure 3. Histopathologic examination findings: The Congo red method was used to detect amyloid in tissue sections. Amyloid was identified as the AA type on immunohistochemical testing with the use of monoclonal antibodies specific to SAA. (A) Hematoxylin and eosin staining of the biopsy specimen, the slightly nodular appearance amorphous, acellular eosinophilic, pale amyloid deposits, expanding the mesangial areas (hematoxylin and eosin, x200). (B) Amyloid gives a characteristic apple-green birefringence when stained with Congo red and viewed under polarized light. There is amyloid deposition in the mesangial area and weakly interstitium (Congo red under polarized light, x200). (C) Mesangia deposits of amyloid reveal positive immunoreactivity with an antibody against amyloid A (anti-AA immunohistochemistry, $\mathrm{x} 200$ ). Amiyloidosis

Table 1. The effect of periodontal therapy on the Inflammatory markers and some laboratory data

\begin{tabular}{|l|c|c|c|c|}
\hline Parameters & $\begin{array}{c}\text { Before } \\
\text { treatment }\end{array}$ & $\begin{array}{c}\text { After } \\
\text { Treatment } \\
\text { Second week }\end{array}$ & Third week & Third month \\
\hline ESR $(\mathrm{mm} / \mathrm{h})$ & 95 & 71 & 68 & 61 \\
\hline WBC $\left(\mathrm{x} 10^{3} \mathrm{ml}\right)$ & 18 & 13.700 & 10.09 & 9.4 \\
\hline Fibrinogen $(\mathrm{mg} / \mathrm{dl})$ & 621 & 364 & 355 & 324 \\
\hline hs-CRP $(\mathrm{mg} / \mathrm{dl})$ & 68.2 & 35.6 & 34.5 & 21.5 \\
\hline Serum total protein $(\mathrm{g} / \mathrm{dl})$ & 3.5 & 3.7 & 3.0 & 3.4 \\
\hline Serum Albumin $(\mathrm{g} / \mathrm{dl})$ & 1 & 1.9 & 2 & 2.01 \\
\hline Serum creatinine & 1.5 & 0.8 & 0.7 & 0.9 \\
\hline Proteinuria (g/day) & 28.500 & 11.447 & 8.65 & 7.40 \\
\hline Ferritin $(\mathrm{ng} / \mathrm{dl})$ & 476 & 256 & 244 & 238 \\
\hline Cholesterol $(\mathrm{mg} / \mathrm{dl})$ & 553 & & & \\
\hline Triglyceride & 574 & & & \\
\hline
\end{tabular}

loss tooth mobility and tooth loss. In addition, several inflammation markers, such as erythrocyte sedimentation rate (ESR), White blood cell count (WBC), fibrinogen and high sensitive C-reactive protein (hs$\mathrm{CRP}$ ) were all elevated. Periodontal diseases are moving into the focus of systemic diseases. Periodontitis is a chronic, long standing and occult infection or inflammation. Sustained overproduction of SAA and other APRs like CRP are prerequisites for the development of systemic AA amyloidosis.

Recently, a systemic review, published by Brunger, et al. [27], a pubMed, Embase and Web of science literature search were performed on causes of AA amyloidosis published in the last four decades. Initially, 4066 unique titles were identified, but only 795 full-text articles and letters were finally selected for analysis. Hundred and fifty diseases were initially reported to be associated with development of AA amyloidosis.
The presence of AA amyloid was proven in 208 articles (26\% of all) of which $140(67 \%)$ showed a strong association with an underlying disease process. Disease associations were categorized and 48 were listed as strong, 19 as weak, 23 as unclear, and 60 as unlikely. Most newly described diseases are not really unexpected because they often cause longstanding inflammation. Based on the spectrum of identified causes, a pragmatic diagnostic approach is proposed for the AA amyloidosis patient in whom an obvious underlying disease is lacking [27]. Identification of the underlying cause provides opportunities for targeted therapy of the inflammatory process, thereby significantly improving the prognosis of the patients [1].

However, in up to approximately $42 \%$ of cases of secondary amyloidosis no underlying etiology can be identified [5-9,27]. Periodontal evaluations are not normally performed as a part of the medical assessment of patients with secondary amyloidosis. Hence, destructive periodontal diseases may be an overlooked source of inflammation in these patients. Periodontal diseases have not been investigated in secondary amyloidosis with unknown etiology.

Although periodontitis and systemic AA amyloidosis have many features in common, to date, only a few reports address the interaction between periodontal disease and secondary amyloidosis [15-17,21]. Three of them [15-17] were published from our center by Cengiz MI, et al.

Study conducted by Cengiz MI, et al. showed that the prevalence of moderate to severe periodontitis in patients with FMF with amyloidosis $(80.6 \%)$ was significantly greater than those in patients with FMF without amyloidosis (38\%) and control patients (20\%). In addition, serum levels of APRs in patients with FMF were reduced significantly following nonsurgical periodontal therapy [15].

In the second study of Cengiz MI, et al. they analyzed the etiological distribution of 112 patients with systemic AA amyloidosis, showed that the prevalence of periodontitis was $47.5 \%$ in patients with FMF, $72.5 \%$ in patients who were either chronically infected or had malignant disease, and $84.6 \%$ in patients with periodontitis. Serum levels of APRs in patients with amyloidosis were reduced significantly after nonsurgical periodontal therapy [16].

The third study of Cengiz MI, et al. was a case report that documented secondary amyloidosis, which was supported by intra-oral and renal biopsies, while ruling out known possible etiological factors as the cause of secondary amyloidosis. The patient developed AA amyloidosis most likely secondary to his long-standing periodontitis. Moreover, this study demonstrated that secondary amyloidosis can be slowed down if periodontal conditions can be improved [17].

The other study conducted by Khoury S, et al. was a case report that illustrated an interaction between systemic amyloidosis and severe periodontitis in a patient with rheumatoid arthritis [21].

Our case looks like the case report that was previously published in our center [15]. But, it was bit different from the other case report [21]. Because results from clinical and epidemiologic studies have suggested that periodontitis and tooth loss are more prevalent in individuals with rheumatoid arthritis.

In this study, our patient's differential diagnosis includes pulmonary $\mathrm{X}$-ray, pulmonary function tests, sputum cytology, and tuberculin skin testing, they were all negative for tuberculosis, and bronchiectasis. His history, physical examination and laboratory findings were negative for rheumatoid arthritis, and FMF patients (rheumatoid factor and MEFV gene mutation were negative, respectively). There was no evidence for chronic infection or inflammation such as systemic rheumatoid 
diseases, other chronic inflammatory arthritis (rheumatoid factor, antinuclear antibody-ANA, antidouble-stranded DNA, anti-neutrophil cytoplasmic antibodies tests) were all negative except chronic periodontitis. There was no clinical evidence for inflammatory bowel diseases or leprosy in our patient.

Secondary amyloidosis is also associated with malignant diseases such as Hodgkin's disease and mesothelioma. Clinical examination abdominal and chest computed tomography were negative for any malignant disorders or airflow obstruction.

A thorough investigation for the etiology that may cause secondary amyloidosis was carried out. None was identified except chronic periodontitis. Our patient presented with typical signs of periodontal disease that include gingival tenderness, bleeding, recession, alveolar bone loss, tooth mobility and tooth loss. In addition, several inflammation markers were all elevated. There was no evidence for chronic infection or inflammation except chronic periodontitis. Our patient developed reactive amyloidosis (AA type), most likely secondary to his long-standing periodontitis. Chronic periodontal disease could exaggerate secondary amyloidosis via increased levels of systemic inflammatory mediators. In addition, our report highlights the possibility that amyloid deposition in patients with systemic amyloidosis causes accelerated periodontal destruction and bone loss of affected teeth. Amyloid deposition within the periodontium elicited on inflammatory reaction similar that of foreign body material. Accelerated destruction of periodontium and associated supporting bone apparently is caused by this foreign-body-type giant cell reaction. Therefore, elimination of local infection associated with periodontal diseases will aid in the reduction of levels of systemic inflammatory mediators, which may slow the progression of secondary amyloidosis.

In this study, patient has macroglossia, moderately diffusely enlarged hepatosplenomegaly; these are common findings in the amyloidosis. But, enlarged appendix was very interesting and unpublished finding in the literature. Enlarged appendix might be associated with amyloid deposition such as other organomegalies that are found in amyloidosis.

In this study, all the results are in accordance with previous reports from our center and the other [15-17,21 respectively]. Our case is the second case report of pure periodontitis-secondary amyloidosis on the third case of the rheumatoid arthritis and periodontitis. The definitive method of diagnosis is tissue biopsy. This may have important implications for the biopsy technique used for the detection of amyloid [28]. If intra-oral biopsies are used more commonly for patients with chronic periodontal disease, amyloid may be found more frequently than expected.

Evidence has suggested that individual genetic susceptibility to amyloidosis may influence the host's response to infection. Nibali, et al. [29] have found the link between polymorphisms of genes encoding for neutrophil receptors and pro-inflammatory cytokines and the presence of pathogenetic bacteria in patients with aggressive periodontitis. The authors then speculated that complex interactions between the microbiota and host genome may be at the basis of patient's susceptibility to aggressive periodontitis. Currently many investigators are trying to define the genotype-phenotype correlations and risk factors for the development of secondary amyloidosis.

\section{Conclusion}

To our knowledge, this is the second case report that documents secondary amyloidosis supported by the intra-oral and kidney biopsies, while ruling out all possible known etiologic factors as a cause for secondary amyloidosis. Periodontitis and chronic diseases such as secondary amyloidosis share many risk factors. The current evidence is such that prevention and treatment of periodontal disease may reduce chronic systemic disease risk at both the individual and community level. We suggest that periodontal evaluation should be performed as a part of the medical assessment of patients with secondary amyloidosis. Further studies in a larger population will provide insight of this rare but destructive systemic disease.

\section{Conflict of interest}

The authors declare that they have no competing interests.

\section{Acknowledgement}

The authors would like to thank Necdet Keleşoğlu, English teacher for his technical support.

\section{Authors contributions}

ÖK followed the patient and collected the data, KC diagnosis, medical treatment, inter disciplines cooperation analyzed and interpreted our patient data, US performed periodontal evaluation, ED collected the data. AÖ, FK and BC performed the histological examination of tissue biopsies. All the authors read and approved the final manuscript.

\section{References}

1. Lachman HJ, Goodman HJB, Gilberkson JA, Gallimore JR, Sabin CA, et al. (2007) Natural history and outcome in systemic AA amyloidosis. N Engl J Med 356: 23612371. [Crossref]

2. Urieli-Shoval S, Linke RP, Matzner Y (2000) Expression and function of serum amyloid A, a majör acute- phase protein, in normal and disease states. Curr Opin Hematol 7: 64-69. [Crossref]

3. Yalçınkaya F, Çakar N, Acar B, Tutar A, Güriş H, et al. (2007) The valve of the levels of acute-phase reactants for the prediction of familial Mediterranean fever associated amyloidosis: a case control study. Rheumatol Int 27: 512-522. [Crossref]

4. Yamada T (1999) Serum amyloid A (SAA): a concise review of biology, assay methods and clinical usefulness. Clin chem lab Med 37: 381-358. [Crossref]

5. Yılmaz M, Unsal A, Sökmen M, Kartanoğulları OH, Alkım C, et al. (2013) Renal involvement in AA amyloidosis: clinical outcomes and survival. Kidney Blood Press Res 37: 33-42.

6. Paydaş S (1999) Report of 59 cases of renal amyloidosis. Int urol Nephrol 31: 619-631. [Crossref]

7. Ahpab E, Kara E, Sahutoğlu T, Baştürk T, Koç Y, et al. (2014) Outcome of 121 patients with renal amyloid a amyloidosis. J Res Med Sci 19: 644-649.

8. Ayar Y, Ersoy A, Öksüz MF, Ocakoğlu G, Vuruşkan BA, et al. (2017) Clinica outcomes and survival in AA amyloidosis Patients. Reu Bras Rheumatol 57: 535-544.

9. Girnius S, Dember L, Doras G, Skinner M (2011) The changing Face of AA amyloidosis: a single center experience. Amyloid 18: 226-228. [Crossref]

10. Waldenström H (1928) On the formation and disappearance of amyloid in man. Acto Chir Scand 63: 479-530.

11. Ebersole JL, Machen RL, Steffen MJ, Willman DE (1997) Systemic acute- phase reactants, C-reactive protein and haptoglobin, in adult periodontitis. Clin exp Immunol 107: 347-352. [Crossref]

12. Taylor BA, Tofler GH, Carey HMR, Morel-Kupp MC, Phitcox S, et al. (2006) Fullmouth tooth extraction lowers systemic inflammatory and thrombotic markers of cardiovascular risk. J Dent Res 85: 74-78.

13. Slade GD, Offenbucher S, Beck JD, Heiss G, Pankow JS (2000) Acute-phase inflammatory response to periodontal disease in US population. J Dent Res 79: 45-47. [Crossref]

14. D'Aiuto F, Nibali L, Parkar M, Suvan J, Tonetti MS (2005) Short-term effects of intensive periodontal therapy on serum inflammatory markers and cholesterol. J Dent Res 84: 269-273. [Crossref] 
15. Cengiz MI, Bağcı H, Cengiz S, Yiğit S, Cengiz K (2008) Periodontal disease in patients with familial Mediterranean fever: From inflammation to amyloidosis. $J$ periodontal Res 44: 354-361. [Crossref]

16. Cengiz MI, Yayla N, Cengiz K, Bağcı H, Taşkın E (2011) Interraction between periodontal disease and systemic secondary amyloidosis: From inflammation to amyloidosis. J periodontol 82: 566-574.

17. Cengiz MI, Wang HL, Yıldız L (2010) Oral involvement in a case of AA amyloidosis: a case report. J Med Case Reports 4: 200-206. [Crossref]

18. Cengiz K (2005) Uncommon etiology in renal amyloidosis. Acta Clinica Belgica 60: 109-113. [Crossref]

19. Nasr SH, Shwars R, D’Agoti VD, Markowitz GS (2006) Paraplegia, proteinuria, and renal failure. Kidney Int 69: 412-415.

20. Glurich I, Grossi S, Albini B, Ho A, Shah R, et al. (2002) Systemic inflammation in cardiovascular and periodontal disease: Comparative study. Clin Diagn Lab Immunol 9: 425-432. [Crossref]

21. Khoury S, Dusek JJ, Anderson GB, Vigneswaran N (2004) Systemic amyloidosis manifesting as localized, severe periodontitis. J Am Dent Assoc 135: 617-623. [Crossref]

22. Agrawal AA, Kabley A, Yeltiwar RK, Purohit HJ (2006) Assessment of single nucleotide polymorphism at IL-1 A+4845 and IL-1 B+3954 as genetic susceptibility test for chronic periodontitis in Maharashtrian ethnicity. J Periodontal 77: 1515-1521.
23. Röcken C, Shakespeare A (2002) Pathology, diagnosis and pathogenesis of AA amyloidosis. Virchows Arch 440: 111-122. [Crossref]

24. Li L, Messas E, Batista EL Jr, Levine RA, Amar S (2002) Porphyromonas gingivalis infection accelerates the progression of atherosclerosis in a heterozygous apolipoprotein E-deficient murine model. Circulation 150: 861-867.

25. Champagne C, Yoshinari N, Oetjen JA, Riche EL, Bleck JD, et al. (2009) Gender differences in systemic inflammation and atheroma formation following porphyromonas gingivalis infection in heterozygous apolipoprotein E-deficient mice. J Periodontal Res 44: 596-577.

26. Ardila CM, Isabel CG (2015) Comparison of serum amyloid A protein and C-reactive protein levels as inflammatory markers in periodontitis. J Periodontal Implant Sci 45: 14-22. [Crossref]

27. Brunger AF, Nienhuis HLA, Bijzet J, Hazenberg BPC (2020) Causes of AA amyloidosis: a systematic review. Amyloid 27: 1-12. [Crossref]

28. Stoopler ET, Sollectio TP, Chen SY (2003) Amyloid deposition in the oral cavity: a retrospective study and review of the literature. Oral Surgery Med Oral Pathol 95: 674-680. [Crossref]

29. Nibali L, Ready DR, Parker M, Brett PM, Wilson M, et al. (2007) Gene polymorphism and prevalence of key periodontal pathogens. J Dent Res 86: 416-420.

Copyright: $₫ 2020$ Kanbolat Ö. This is an open-access article distributed under the terms of the Creative Commons Attribution License, which permits unrestricted use, distribution, and reproduction in any medium, provided the original author and source are credited. 